\title{
Unconditional jetting
}

\author{
Alfonso M. Gañán-Calvo \\ ESI, Universidad de Sevilla, Camino de los Descubrimientos, s/n 41092, Spain \\ (Received 6 April 2008; revised manuscript received 8 June 2008; published 11 August 2008)
}

\begin{abstract}
Capillary jetting of a fluid dispersed into another immiscible phase is usually limited by a critical capillary number, a function of the Reynolds number and the fluid property ratios. Critical conditions are set when the minimum spreading velocity of small perturbations $v^{*}$ along the jet (marginal stability velocity) is zero. Here we identify and describe parametric regions of high technological relevance, where $v^{*}>0$ and the jet flow is always supercritical independently of the dispersed liquid flow rate; within these relatively broad regions, the jet does not undergo the usual dripping-jetting transition, so that either the jet can be made arbitrarily thin (yielding droplets of any imaginably small size), or the issuing flow rate can be made arbitrarily small. In this work, we provide illustrative analytical studies of asymptotic cases for both negligible and dominant inertia forces. In this latter case, requiring a nonzero jet surface velocity, axisymmetric perturbation waves "surf" downstream for all given wave numbers, while the liquid bulk can remain static. In the former case (implying small Reynolds flow) we found that the jet profile small slope is limited by a critical value; different published experiments support our predictions.
\end{abstract}

DOI: 10.1103/PhysRevE.78.026304

PACS number(s): 47.55.db, 47.20.Dr, 47.65.- d

\section{INTRODUCTORY REMARKS}

The quest for the conditions under which a given stream of fluid 1 can be dispersed as very small, homogeneously sized droplets into another immiscible fluid 2 is an old endeavor. Steady capillary jetting produces droplets of any desired diameter at a controllable rate through the RayleighPlateau instability, and thus is the preferred choice in many applications. Jetting can be supported by a diversity of energy sources, from plain pressure [1] or electrostatic suction [2] (or their combination [3,4]), to chemical potential [5], or even thermal gradients. Very recently, jetting has also been shown to take place under concentrated photon irradiation (laser) when surface tension is extremely low [6]. Capillary jetting from a fluid source gives rise to droplets smaller than dripping (a phenomenon where drops are individually issued from the source at a certain frequency) under the same operating conditions. Consequently, a significant effort has been lately devoted to map the transition from jetting to dripping from a fluid source [7-10], in the search for extended jetting conditions down to the smallest possible jet diameter. Two key dimensionless numbers gauge the role of inertia and viscous forces relative to surface tension, namely, Weber and capillary numbers $\mathrm{We}=\rho_{1} U_{s}^{2} R / \sigma$ and $\mathrm{Ca}=\mu_{1} U_{s} / \sigma, \rho_{1}, \mu_{1}$, and $\sigma$ being the density, viscosity, and surface tension of the dispersed fluid. $U_{s}$ and $R$ are the jet surface velocity and radius, respectively. Alternatively, the Reynolds number $\mathrm{Re}$ $=\mathrm{We} / \mathrm{Ca}=\rho_{1} U_{s} d / \mu_{1}$ and $\mathrm{Ca}$ can be used to characterize the jet dynamics; this choice is particularly useful in microfluidics, where Re is usually moderate or small (laminar flows). Surface tension is the main agent sustaining wave propagation of disturbances along the jet (downstream coordinate $z$ ), and thus $\mathrm{Ca}$ is the key parameter controlling the jet dynamics in microfluidics when Re is small.

The link connecting convective and absolute instability to jetting and dripping, respectively, is well documented by experiments [7-10]. Thus, the rate at which the perturbations grow is a function of $\mathrm{Re}$ and $\mathrm{Ca}$. Since the jet disperses fragments of fluid 1 into an immiscible jet 2 of density $\rho_{2}$ and viscosity $\mu_{2}$, two additional parameters are the fluid density and viscosity ratios $\rho=\rho_{2} / \rho_{1}$ and $\mu=\mu_{2} / \mu_{1}$. When jetting is produced by flow focusing [11], one has a stream of fluid 2 forced through an orifice of diameter $D$ which focuses the jet of fluid 1 (see Fig. 1, inset). In flow focusing, we assume that $R \ll D$, the most usual case. The relationship between the axial velocity $U_{z}$ of fluid 2 near the axis of the exit orifice, $U_{z}=U_{2}$, and the flow rate of fluid 2 through the orifice, $Q_{2}$, depends on the Reynolds number $\operatorname{Re}_{D}$ $=\rho_{2} Q_{2} /\left(\mu_{2} D\right)$ (see Fig. 1). For $\operatorname{Re}_{D} \rightarrow \infty$, one asymptotically has [12] $U_{2}=0.5 \bar{U}_{2}$, where $\bar{U}_{2}=4 Q_{2} /\left(\pi D^{2}\right)$. However, for $\operatorname{Re}_{D} \ll 1$, one approximately has $U_{2} \simeq 2 \bar{U}_{2}$. For moderate to high $\operatorname{Re}_{D}$, a useful approximation is $U_{2} \sim \bar{U}_{2}$ (see Fig. 1 for $\left.\mathrm{Re}_{D}=2200\right)$. In addition when viscous effects dominate in the flow of fluid 1 , the jet dynamics is thus determined by the

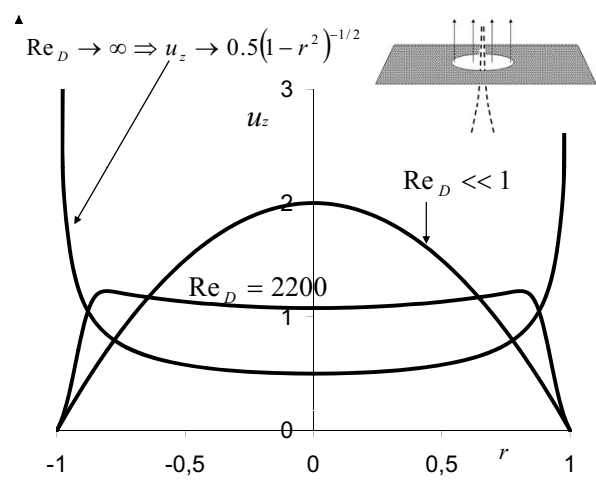

FIG. 1. Axial velocity profile $u_{z}=U_{z} / \bar{U}_{2}$ as a function of the radial coordinate $r$ (made nondimensional with $D / 2$ ) for an incompressible fluid at a round orifice in an infinite plane thin wall as a function of the radial coordinate for various $\operatorname{Re}_{D}$ values. The flow is forced through the orifice by a pressure difference across the thin wall. In particular, the case $\operatorname{Re}_{D}=2200$ has been obtained using numerical simulation (volumes of fluid). The inset shows the basic flow configuration. 


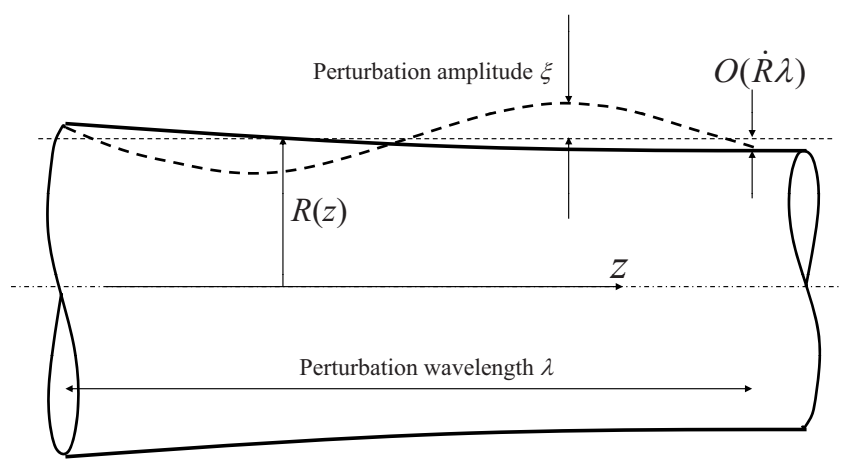

FIG. 2. Sketch of a perturbed jet portion.

parameters $\{\mathrm{Ca}, \rho, \mu\}$ only, since Re disappears from the analysis. In the limit $\operatorname{Re} \rightarrow \infty, \operatorname{Re}$ is out from the analysis as well, and viscous effects are confined to boundary layers at the interface [13]. Since in this case the jet radius $R$ is implicitly given by the equation $\rho_{2} U_{2}^{2}=2 \sigma / R+\rho_{1} U_{1}^{2}$, the jet dynamics becomes governed by $\{\mathrm{We}, \rho, \mu, U\}$, where $U=U_{2} / U_{1}$ and $U_{1}=Q_{1} /\left(\pi R^{2}\right)\left(Q_{1}\right.$ is the issuing liquid flow rate of fluid 1). These two limiting cases allow for analytical treatment, and will be discussed in this work.

Naturally, a jet is characterized by its slenderness and the applicability of the usual slender flow approximations. To study the linear stability of the system, we interrogate the jet response to small perturbation amplitudes $\xi \ll R$ with a given axial wavelength $\lambda$ (see Fig. 2). As long as the jet curvature is very approximately $R^{-1}(z)$, and the jet radius variations along axial distances $\lambda$, of the order of $O(\dot{R} \lambda)$ (where $\dot{R}$ $=d R / d z$ ), are small compared to $R$, one can always choose amplitudes such that $O(\dot{R} \lambda) \ll O(\xi) \ll O(R)$, which justifies the classical cylindrical approximation. Under these assumptions, we investigate the linear dynamics of cylindrical steady capillary jets in two limits: (i) creeping flow limit $(\operatorname{Re} \rightarrow 0)$, and (ii) inertia-dominated limit (large Re). In the first limit, our exploration will focus on some practical scenarios of jetting at very small scales very recently reported in the literature [10,14-18].

Consider a thin cylindrical jet of fluid 1, moving in a coflowing fluid 2. The general capillary flow category claimed in this work, for which the flow rate $Q_{1}$ could be made arbitrarily small (i.e., jetting would always take place independently of the issuing flow rate), requires either making the jet radius $R$ very small or allowing for recirculation (part of the jet inner flow should flow upstream) for continuity. Asymptotically, one may as well have a static jet core $\left(U_{1}=0\right)$ surrounded by a coflowing fluid with velocity $U_{2}$, which demands a negligible viscous diffusion from the interface. In this work, we will study these configurations which allow analytic dispersion relations. Thus, the occurrence of unconditional jetting (UJ) is not restricted to either small or large Re values; in fact, the only necessary condition is that the jet surface velocity should be large enough to convect all perturbations downstream. The limit cases of large and small Re will be discussed in some detail in this work because they allow for analytical treatment and for their importance and illustrative power. In this sense, given the utter importance of controlled micrometer- and nano-sized droplet generation, (a)

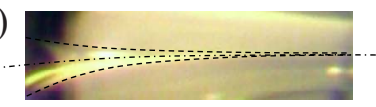

(b)

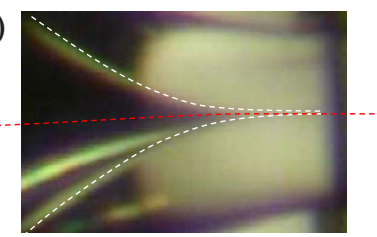

(c)

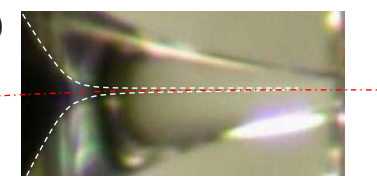

FIG. 3. (Color online) Different coflowing capillary jets into a much thicker silicone oil (which is in turn focused by an outer gas flow), from Ref. [14]: (a) an air jet $\left(\mu=2.06 \times 10^{4}\right)$; (b) a mercury jet $\left(\mu=2.42 \times 10^{2}\right)$; (c) an ink solution jet $(\mu=22.2)$. The lines guide the eye along the jet interface. Dash-dotted lines approximately delineate the positions of the centers of the cross section area, not in a straight line owing to a slight bend of the outer flow. Observe that the overall jet slope increases as the viscosity ratio $\mu$ decreases.

we aim to provide a global understanding of mechanisms supporting UJ, to guide future fluid disperser designs of special relevance in chemical engineering, combustion and energy efficiency, transport, food processing, spraying, biochemistry, pharmacy, biomedicine, and environmental engineering, among others.

A steady capillary jet of a fluid surrounded by an immiscible continuum fluid phase (Fig. 3), an intrinsically unstable state [19], is locally stable (jetting is possible) whenever the so called marginal stability velocity $v_{-}^{*}$ relative to an observer is positive, so that all perturbations are convected downstream. From now on, whenever we assume a local analysis applicable, velocities are made nondimensional with the local surface velocity $U_{s}$. Since dripping is axisymmetric, here we consider axisymmetric perturbations only. Assuming small perturbations as superposition of waves proportional to $\exp [i(k z-\omega t)]$, where wave number $k=k_{r}+i k_{i}$ and frequency $\omega=\omega_{r}+i \omega_{i}$ (made nondimensional with $R^{-1}$ and $U_{s} / R$, respectively) are complex numbers, the jet linear dynamics is governed by the dispersion relation between $k$ and $\omega$. Central to our analysis is the fact that steady capillary jets are unstable states $[19,20]$, that is, they exhibit wave number ranges $k$ such that perturbations grow in time as $\exp \left(\omega_{i} t\right)$ leading to break up. A growing disturbance usually spreads along the jet bounded by two fronts moving with velocities $v_{+}^{*}$ and $v_{-}^{*}$, the extremal values of the envelope velocities $v$ $=\omega_{i} / k_{i}[19-22]$. The extremal values or marginal stability velocities $v^{*}$ should satisfy

$$
v^{*}=\omega_{i}^{*} / k_{i}^{*}=\partial \omega_{i} /\left.\partial k_{i}\right|_{k=k^{*},}, \quad \partial \omega_{i} /\left.\partial k_{r}\right|_{k=k^{*}}=0 .
$$

Thus, the fate of the jet at a source-fixed station is determined by the minimum marginal stability velocity $v_{-}^{*}$. If $v_{-}^{*}>0$, small perturbations are convected downstream for all wave numbers (convective instability, or local stability), 
while if $v_{-}^{*}<0$, some wave number ranges will grow locally without bound (absolute instability). The aim of this work is to report a special class of parametric conditions of capillary jetting for which the marginal stability velocity $v_{-}^{*}$ (minimum front propagation velocity) remains always positive for vanishing dispersed flow rates of fluid 1 . We designate this rather singular flow condition unconditional jetting. This means that the capillary jet is convectively unstable, or locally stable, and does not undergo a jetting-dripping transition as the issuing flow rate $Q_{1}$ vanishes. The technological relevance of this class of flows can be understood as follows: picture a steady capillary jet flowing down from a slightly opened tap. If $v_{-}^{*}$ were always positive, one could slowly turn off the tap without transition to dripping. Eventually, when the tap is turned off, the jet would thin down to the continuum limit without transition to dripping. For extremely small flow rates, nearly monodisperse droplets of any imaginably small size would be produced upon Rayleigh breakup at a highly controllable rate. Although this behavior is indeed rather unusual for laminar jets from taps, it is however a real occurrence in coflowing jets for a certain rather ample ratios of continuous-dispersed fluid densities and viscosities [4] $\rho$ and $\mu$, or when the continuous phase 2 coflows with the jet at a velocity larger than a critical velocity $[14,13] u_{2}^{*}$. We will see that the fundamental physical requirement for $\mathrm{UJ}$ is to have a jet surface velocity above a critical one.

\section{THE CASE OF NEGLIGIBLE INERTIA: ULTRA-THIN JETTING}

First, we will consider the case where the velocities of both fluids are equal to the surface velocity (flat velocity profiles). Here, the jet's linear dynamics is governed by the following dispersion relation $[9,23]$ :

$$
i \operatorname{Ca}(\omega-k)\left(\frac{N(k, \omega, \operatorname{Re}, \rho, \mu)}{D(k, \omega, \operatorname{Re}, \rho, \mu)}+2(1-\mu)\right)+\left(k^{2}-1\right)=0 .
$$

For convenience, we define "viscous" wave numbers for both fluids as

$$
k_{1}^{2}=k^{2}-i \operatorname{Re}(\omega-k), \quad k_{2}^{2}=k^{2}-i \rho \mu^{-1} \operatorname{Re}(\omega-k) .
$$

Using these definitions, functions $N$ and $D$ are expressed as

$$
\begin{aligned}
N \equiv & 2 k \mu k_{1} k_{2}\left[K_{0}\left(k_{2}\right) I_{1}\left(k_{1}\right) k_{1}+I_{0}\left(k_{1}\right) K_{1}\left(k_{2}\right) k_{2}\right]+k\left[k^{2}(\mu-1)\right. \\
& \left.-k_{1}^{2}+\mu k_{2}^{2}\right]^{2} I_{0}(k) I_{1}\left(k_{1}\right) K_{0}(k) K_{1}\left(k_{2}\right)+4 k^{3} k_{1} k_{2}(\mu \\
& -1)^{2} I_{0}\left(k_{1}\right) I_{1}(k) K_{0}\left(k_{2}\right) K_{1}(k)-k_{2} I_{1}\left(k_{1}\right) K_{0}\left(k_{2}\right)\left\{\left[k^{4}+k_{1}^{2} k_{2}^{2}\right.\right. \\
& \left.+k^{2}\left(k_{1}^{2}-k_{2}^{2}\right)\right] \mu I_{1}(k) K_{0}(k)+\left[k_{1}^{4}+k^{4}(1-2 \mu)^{2}-2 k^{2} k_{1}^{2}(\mu\right. \\
& \left.-1)] I_{0}(k) K_{1}(k)\right\}-k_{1} I_{0}\left(k_{1}\right) K_{1}\left(k_{2}\right)\left\{\left[k^{4}(\mu-2)^{2}\right.\right. \\
& \left.+2 k^{2} k_{2}^{2} \mu(\mu-1)+\mu^{2} k_{2}^{4}\right] I_{1}(k) K_{0}(k)+\left[k^{2}\left(k^{2}-k_{1}^{2}\right)+k_{2}^{2}\left(k^{2}\right.\right. \\
& \left.\left.\left.+k_{1}^{2}\right)\right] \mu I_{0}(k) K_{1}(k)\right\}, \\
D \equiv & k\left\{\left[k_{2} K_{0}\left(k_{2}\right) K_{1}(k)-k K_{0}(k) K_{1}\left(k_{2}\right)\right]\left(k_{1}^{2}-k^{2}\right) I_{1}(k) I_{1}\left(k_{1}\right)\right. \\
& \left.+\mu\left[k_{1} I_{0}\left(k_{1}\right) I_{1}(k)-k I_{0}(k) I_{1}\left(k_{1}\right)\right]\left(k_{2}^{2}-k^{2}\right) K_{1}(k) K_{1}\left(k_{2}\right)\right\} .
\end{aligned}
$$

Interestingly, a series expansion of $N$ and $D$ around $\mathrm{Re}=0$ yields

$$
\begin{aligned}
N & =\frac{(\omega-k)^{2}}{4 k} N_{2}(k, \mu) \operatorname{Re}^{2}+O\left(\operatorname{Re}^{3}\right), \quad D \\
& =\frac{(\omega-k)^{2}}{4 \mu k} D_{2}(k, \mu) \operatorname{Re}^{2}+O\left(\operatorname{Re}^{3}\right),
\end{aligned}
$$

where $N_{2}$ and $D_{2}$ (omitted, lengthy expressions) are independent of $\omega$ and $\rho$, as can be checked using MATHEMATICA. Moreover, in this limit the unperturbed fluid velocities do not need to be uniform. Defining for convenience an average capillary number as

$$
\overline{\mathrm{Ca}}=\mu^{1 / 2} \mathrm{Ca}=\left(\mu_{1} \mu_{2}\right)^{1 / 2} U_{s} / \sigma,
$$

Eq. (2) yields an explicit analytical expression for $\omega$ $=\omega(k, \overline{\mathrm{Ca}}, \mu)$ providing a closed form for the frequency $\omega$ :

$$
\omega=k+i\left(k^{2}-1\right) \overline{\mathrm{Ca}}^{-1}\left(\mu^{1 / 2} \frac{N_{2}(k, \mu)}{D_{2}(k, \mu)}+2\left(\mu^{-1 / 2}-\mu^{1 / 2}\right)\right)^{-1} .
$$

This equation, together with conditions (1), provides $v^{*}, \omega^{*}$, and $k^{*}$ for a given set $\{\overline{\mathrm{Ca}}, \mu\}$. Since $\overline{\mathrm{Ca}}$ does not depend on the jet diameter $d$, a particularly strong practical implication follows: if one can find a parametric region $\{\overline{\mathrm{Ca}}, \mu\}$ where $v^{*}$ is always positive, it is so for any value of the jet diameter, no matter how small it can be.

Figure 4 shows a plot of the loci $\overline{\mathrm{Ca}}=\overline{\mathrm{Ca}} *(\mu)$ where $v^{*}$ $=0$. Fifteen orders of magnitude in $\mu$ are explored, showing a small dependency of the critical $\overline{\mathrm{Ca}}$ on $\mu$, which supports our definition choice for a relevant capillary number in the creeping flow limit [jet radii $R \ll \mu_{1} /\left(\rho_{1} U_{s}\right)$ ], which incorporates both inner and outer fluid viscosities. This curve splits the $\{\mu, \overline{\mathrm{Ca}}\}$ plane into two halves: above (below) this curve, $v_{-}^{*}$ is always positive (negative) and the jet flow is always supercritical (subcritical) independently of the jet diameter (when supercritical, the convective velocity always overcomes the upstream spreading of perturbations). Thus, if the unperturbed velocity profile of both fluids is homogeneous in and out of the jet, supercritical jets of any imaginably small diameter could be produced for a coflow speed $u_{s}$ larger than a critical velocity $u_{s}^{*}=\overline{\mathrm{Ca}}^{*}\left[U_{s}^{*}=\sigma \overline{\mathrm{Ca}}^{*} /\left(\mu_{1} \mu_{2}\right)^{1 / 2}\right]$.

Experimental results of other authors are compared with theory in Fig. 4. Anna et al. [15] used a planar flow focusing device where they dispersed water $\left(\mu_{1}=1 \mathrm{mPa} \mathrm{s}\right.$, $\left.\rho_{1}=1 \mathrm{~kg} \mathrm{l}^{-1}\right)$ in silicone oil $\left(\mu_{2}=5 \mathrm{mPa} \mathrm{s}, \rho_{2} \simeq 0.9 \mathrm{~kg} \mathrm{l}^{-1}\right)$. Their experiments show (Anna et al. [15]), Figs. 3(e), 3(k), and $3(\mathrm{q})$, that jetting was found for values of the focusing oil flow rate $Q_{2}=4.2 \mu \mathrm{s} \mathrm{s}^{-1}$ and above. Since their Reynolds number at the orifice was about 6 , a calculation of the oil velocity at the orifice axis yields about $U_{2}=2 Q_{0} /(h D)$ $=1.65 \mathrm{~m} / \mathrm{s}$, where $h=117 \mu \mathrm{m}$ and $D=43.5 \mu \mathrm{m}$ are the orifice depth and width, respectively (their orifice length was about $L=120 \mu \mathrm{m}$ from their pictures, and thus a parabolic velocity profile should have developed). In accord with our 


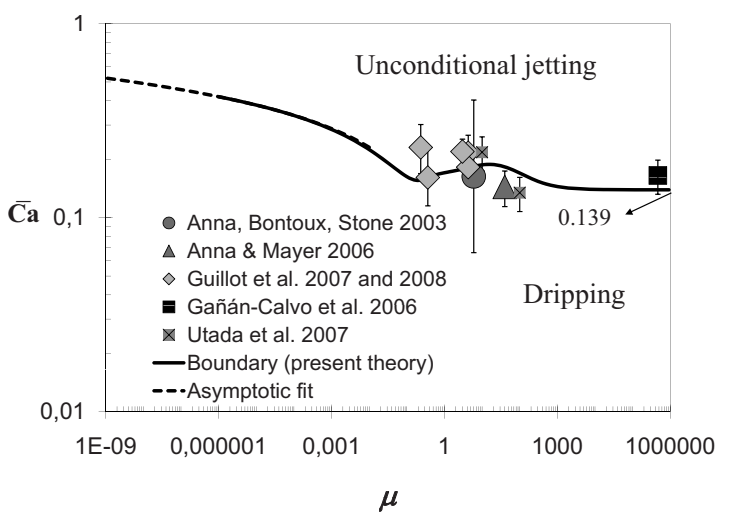

FIG. 4. Critical capillary number as a function of the viscosity ratio $\mu$. An asymptotic fit for $\mu \ll 1$ is provided: $\overline{\mathrm{Ca}}^{*}$ $\rightarrow 0.1[\ln (\mu)]^{0.5465}$. Also given: comparison with published experimental results where conditions for observed dripping-jetting transition are recorded, assuming a homogeneous velocity profile of both fluids in and out of the jet. Unless otherwise stated, the estimated errors associated with data extraction from published plots are about $\pm 20 \%$. Data taken from (symbols, authors, data source figures): $\bigcirc$ Anna et al. [15], Fig. 3; $\triangle$ Anna and Mayer [18], Fig. 5a; $\times$ Utada et al. [24], Fig. 4; $\diamond$ Guillot et al. [10], Figs. 5(a1) and 5(a2), and Herrada, Gañán-Calvo, and Guillot [25]. Fig. 3; $\square$ Gañán-Calvo et al. [23], Fig. 4. (Liquid surface tension was approximately $50 \mathrm{mN} / \mathrm{m}$; liquid velocity at the orifice entrance can be calculated approximately [26] as $U=k \Delta P D / \mu_{2}$, where $\Delta P$ is the pressure drop through the orifice, and $k$ is about 0.5.) In particular, for Anna et al. [15], $\mu_{1}=0.001 \mathrm{~Pa} \mathrm{~s}, \mu_{2}=0.006 \mathrm{~Pa} \mathrm{~s}, 0.005<\sigma$ $<0.01 \mathrm{~N} / \mathrm{m}$ [27], $1.4<Q_{2}<4.2 \mu \mathrm{l} / \mathrm{s}$ or $0.27<U_{2}<0.83 \mathrm{~m} / \mathrm{s}$, which gives $0.066<\overline{\mathrm{Ca}}<0.404$ in this case.

predictions, they found jetting to occur above the indicated oil flow rate independently of the oil-water flow rate ratio, i.e., independently of how thin the jet was. Their corresponding threshold $\overline{\mathrm{Ca}}=\left(\mu_{1} \mu_{2}\right)^{1 / 2} U_{2} / \sigma$ is about 0.169 (with errors associated with surface tension [27] and indetermination between $Q_{0}=1.4$ and $4.2 \mu \mathrm{l} / \mathrm{s}$ ). In addition, using their same planar flow focusing device, Anna and Mayer [18] recently reported jetting independently of the focused flow rate beyond a capillary number $\overline{\mathrm{Ca}}=0.144$ (worked out from their disclosed data), for an aqueous solution focused by oil with $\mu=40$, in the absence of surfactants. They reported transition from dripping to jetting for outer-to-inner flow rate ratios as large as 300, corresponding to jet diameters as small as about $3 \mu \mathrm{m}$. When surfactants are present, their results cannot be compared owing to nonlinear dynamic surface tension effects beyond the critical micelle concentration (as they declare) at the jet.

Moreover, Guillot et al. [10] have reported an extensive and very valuable series of experiments of a liquid jet flowing coaxially in another immiscible liquid inside a cylindrical channel. When the jet to channel diameter ratio becomes very small, their measurements can be compared to our predictions. Their results show the dripping to jetting transition for a viscosity ratio $\mu=4.3$ at $\overline{\mathrm{Ca}}=0.18$ and 0.22 (calculated from their published data in their Figs. a1 and a2, respectively, for their higher available outer-to-inner flow rate ratios). Other experimental results by Guillot (in [25]) are also

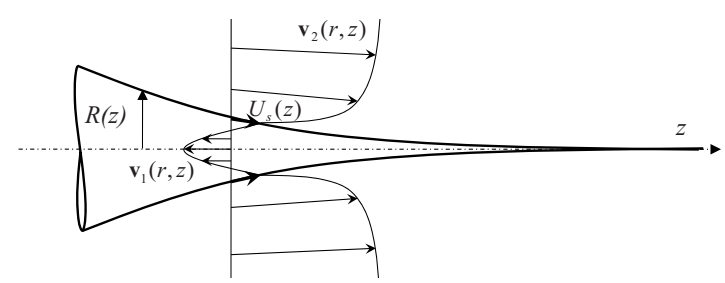

FIG. 5. Sketch of a jet tapering from a finite-sized source in the form of an arbitrarily thin spout. In this configuration, the fluid velocities cannot be homogeneous for conservation of mass.

shown. We do not make use of their results with surfactants for the same reasons given above about results from Anna and Mayer [18]. Finally, for very large outer-to-inner viscosity fluid ratio, $\mu=4.7 \times 10^{5}$ (syrup-air), we have found [23] transition from bubbling to jetting at $\overline{\mathrm{Ca}} \simeq 0.18$. All these experimental findings, plotted in Fig. 4, provide full support to our prediction for homogeneous (flat) velocity profiles.

Furthermore, we will consider a limit situation where the jet tapers from a finitely sized source (e.g., a capillary tube of inner radius $R_{0}$ ), forming an arbitrarily thin spout of fluid 1 (Fig. 5). For ease of understanding in this case, where we need to consider axial dependency of jet radius and velocities, we turn to dimensional expressions. Here, the local unperturbed velocity profiles of fluids for negligible inertia, satisfying stress balance at the jet's surface, are given by:

$$
\begin{gathered}
U_{1}(r, z)=U_{s}(z)+\frac{\sigma \dot{R}(z)}{4 \mu_{1}}\left[1-\left(\frac{r}{R(z)}\right)^{2}\right], \\
U_{2}(r, z)=U_{s}(z)-\frac{\sigma \dot{R}(z)}{2 \mu_{2}} \ln \left(\frac{r}{R(z)}\right) .
\end{gathered}
$$

To obtain these approximate equations, we have assumed that (i) the jet is slender ( $\dot{R}$ is sufficiently small) and transversal velocities are neglected, and (ii) the outer pressure becomes negligible compared to the inner jet pressure $p_{1}$ $\simeq \sigma / R(z)$ as the spout radius becomes very small. Now, mass continuity for a vanishing issuing flow rate of fluid $1, Q_{1}$ $\simeq 0$, yields

$$
U_{s}(z)=\frac{-\sigma \dot{R}(z)}{8 \mu_{1}} .
$$

Substituting this value of the surface velocity in expression (7) for the average capillary number, one has that the jet will be locally stable if its local slope satisfies:

$$
-\dot{R}<\frac{8 \overline{\mathrm{Ca}^{*}}}{\mu^{1 / 2}} .
$$

This limiting slope is only a function of the viscosity ratio $\mu$, plotted in Fig. 6. To obtain the function $\dot{R}^{*}=\dot{R}^{*}(\mu)$, we note that the critical capillary number is independent of the velocity profile shape when $\operatorname{Re} \ll 1$, plugging in the values of the critical capillary number of Fig. 4. This reveals that the jet slope is limited by the inverse of the viscosity ratio $\mu$ only, once the flow of both fluids is dominated by viscosity. It is worth noting that viscosity ratios smaller than about 0.01 


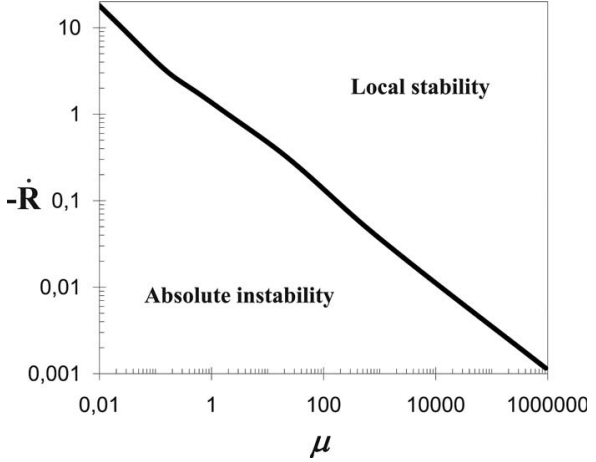

FIG. 6. Limiting slope of the jet as a function of the viscosity ratio $\mu$.

will always provide, for $\mathrm{Re} \ll 1$, a local stability of the tapering meniscus if the flow configuration allows coflowing speeds $U_{s}$ larger than $\sigma \overline{\mathrm{Ca}}^{*} /\left(\mu_{1} \mu_{2}\right)^{1 / 2}$, once jet is issued.

The latter result, asymptotically valid for $\dot{R}$ small, is of importance to at least qualitatively explain experimental observations. In fact, if one observes Fig. 3, the overall critical spout slope decreases as the viscosity ratio $\mu$ increases, according to equation (11). It explains why gas spouts are extremely difficult to achieve in coflowing liquids $(\mu \gg 1)$, since the local requirement (11) becomes very difficult to satisfy unless a very small gas source is used. Nevertheless, upstream of the tapering thin spout, when the meniscus slope becomes of the order unity, the local Reynolds number may not be necessarily small and the limiting conditions for local stability deviate significantly from the above requirements. In fact, the critical capillary number decreases when Re increases (see Montanero and Gañán-Calvo [28], Fig. 5), and therefore the critical slope may increase, becoming of the order unity, in accord with real configurations like the ones shown in Fig. 3. Notwithstanding this, again, the requirement (11) gives at least a qualitative explanation of observations on the slopes of tapering spouts and the difficulty of obtaining stable gas spouts.

A remark on the experiments by Courrech du Pont and Eggers [17] is necessary here: these authors report a "universal" critical value of the slope $\dot{R}=0.47 \pm 0.06$ of a tip singularity in a viscous combined withdrawal of air through a small orifice by means of a viscous liquid, a silicone oil with $\mu_{2}=30$ or $60 \mathrm{~Pa} \mathrm{~s}$. This remarkable result, from a large set of experiments, points to the existence of a locally self-similar conical region which eventually should tapper into an arbitrarily thin gas spout, where condition (11) should hold once the gas flow becomes viscosity dominated in the spout. That self-similar region (we do not deal with this problem here) should be a function of the viscosity ratio $\mu$ only, although the structure of that locally self-similar flow is not known yet. However, our theory predicts critical slopes (about 6 $\times 10^{-4}$ ) much smaller than the one reported in [17], should a sustained (steady) thin spout be formed. Here we propose that the flow observed in [17] does not actually yield a steady extremely thin jet downstream of the observed region, within the suction orifice, for the experimental conditions tested. In fact, these authors report that their setup "fails to produce a thin spout," and that visible bubbles appear downstream of the orifice when entrainment takes place. The entrained spout would only be locally stable down to any imaginably small scale, leading to bubble sizes comparable to the spout diameter, if they had a local velocity at the orifice larger than 0.312 or $0.441 \mathrm{~m} / \mathrm{s}$ for their experimental conditions $\left(\mu_{2}\right.$ $=60$ or $30 \mathrm{~Pa} \mathrm{~s}$, respectively, with $\sigma=0.0213 \mathrm{~N} / \mathrm{m}$ and $\left.\overline{\mathrm{Ca}}^{*}=0.139\right)$. Since their velocities were below $0.0254 \mathrm{~m} / \mathrm{s}$ (velocity at the axis corresponding to a flow rate of $0.01 \mathrm{ml} / \mathrm{s}$ through an orifice of $1 \mathrm{~mm}$ for viscositydominated flow) they could not form a sustained extremely thin spout according to our prediction. However, the molecular mean free path for air enters into play below $1 \mu \mathrm{m}$ [17], and our theory becomes questionable below that scale.

Finally, the elegant mathematical solution for a steady thin spout of Zhang [29] would be stable if her local convective velocities at the spout were everywhere larger than the ones predicted here. This condition would add an extra requirement in her analysis.

The implication of the present conclusions in microfluidics and, in particular, in the field of device design for emulsification are highly attractive for technological applications.

\section{A CASE WITH DOMINANT INERTIA}

While the above analysis is restricted to negligible inertia, in the limit of dominant inertia one can also find an interesting, analytically tractable asymptotic case of UJ [4], i.e., where the issued flow rate $Q_{1}$ can be made arbitrarily small as well. In this alternative case, viscous effects are confined to thin boundary layers at both sides of the jet surface, where bulk velocities $U_{1}$ and $U_{2}$ are nearly flat but different. If both layers develop simultaneously from the same station near the dispersed fluid source, while they are thin compared to the jet diameter the surface velocity $U_{s}$ can be explicitly expressed as (see the arguments given in [4])

$$
u_{s}=U_{s} / U_{1}=\frac{1+(\rho \mu)^{1 / 3} U}{1+(\rho \mu)^{1 / 3}},
$$

where, again, $U=U_{2} / U_{1}$. In this situation, if boundary layers are sufficiently small compared to the jet radius, the dispersion relation for an infinite cylindrical capillary jet moving with bulk velocity $U_{1}$ in a coflowing fluid with bulk velocity $U_{2}$ is

$$
\begin{aligned}
& (\omega-k)\left(\omega-k u_{s}\right) \frac{I_{0}(k)}{I_{1}(k)}+\rho\left(\omega-k u_{s}\right)(\omega-k U) \frac{K_{0}(k)}{K_{1}(k)} \\
& =\frac{\rho U^{2} k\left(k^{2}-1\right)}{\mathrm{We}_{2}},
\end{aligned}
$$

where $\mathrm{We}_{2}=\rho_{2} U_{2}^{2} d /(2 \sigma)$. Note that the Reynolds number is absent in this expression, consistently with the assumption of dominant inertia. Viscous effects are, however, important at the jet surface, making $\mu$ an essential parameter through the jet surface velocity $u_{s}=u_{s}(U, \rho, \mu) \neq 1$. If we seek conditions yielding small dispersed flow rates, i.e., $U \rightarrow \infty$, Eq. (13) plus conditions (7) with $v_{-}^{*}=0$ yields the critical value $\mathrm{We}_{2}^{*}$ using expansions $\omega=\omega_{0}+\bar{U}^{-1} \omega_{1}$ and $k=k_{0}+U^{-1} k_{1}$ (see [30]). Some 


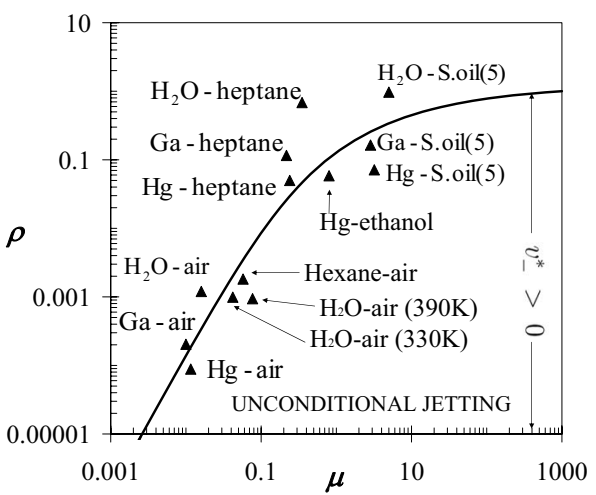

FIG. 7. Parametric region in the $\{\mu, \rho\}$ space where unconditional jetting is found for flow focused jets. Thirteen fluid combinations of interest are shown. Interestingly, water focused by air can theoretically exhibit unconditional jetting for ambient temperatures above $320 \mathrm{~K}$.

flow configurations, such as flow focusing, give rise to further constraints; for very low viscosities, equilibrium requires $\mathrm{We}_{2} \rightarrow 2$ for $U \rightarrow \infty$ (from the equation $\rho_{2} U_{2}^{2}=2 \sigma / R$ $\left.+\rho_{1} U_{1}^{2}\right)$. In this case, for a given density ratio $\rho$, supercritical conditions are found for continuous phase viscosity larger than a critical ratio over the dispersed phase viscosity, or when $\rho$ is smaller than a critical ratio for a given $\mu$ value, as shown in Fig. 7 (see also [4], Fig. 3) where 13 possible flow focusing fluid combinations are plotted. Note that the points correspond to given fluid properties, not reflecting transitions: the fluid combinations in the region "unconditional jetting" would exhibit this behavior, while those outside that region would show a dripping-jetting transition only for a nonzero flow rate $Q_{1}$. In other words, for the points in the region of UJ in Fig. 7, surface velocities are always large enough to have $v_{-}^{*}>0$ and perturbations are flushed downstream, even though the bulk fluid can be literally static.

\section{CONCLUDING REMARKS}

Interestingly enough, upon consideration of Eq. 8 in [4], the limit $U \rightarrow \infty$ considered here is strictly valid independently of the jet electrification as well, since the electrical term (finite) is overcome by other terms proportional to $U$ $\gg 1$ and $U^{2}$. Obviously, viscous diffusion of momentum from their surface soon affect the entire cross section of these jets. From well known boundary layer analysis, the downstream axial length $L_{\mu}$ that must be traveled to achieve $\delta_{1} \sim d$ should satisfy $\left(\mu_{1} L_{\mu} \rho_{1}^{-1} U_{s}^{-1}\right)^{1 / 2} \sim \delta_{1} \sim d$. Thus, using Eq. (12), the maximum "unconditionally stable" length $L_{\mu}$ is limited to

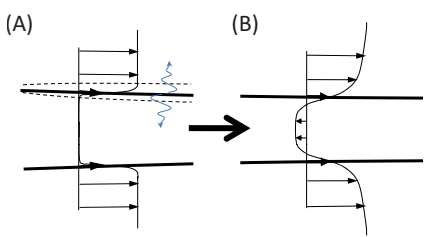

FIG. 8. (Color online) Illustrative sketch of the UJ case for dominant inertia. (a) Flow within length $L_{\mu}$ from the jet source, where the asymptotic analysis is valid but viscous momentum diffusion from the surface thickens the boundary layers. (b) Development of a recirculation pattern downstream of the length $L_{\mu}$ owing to viscous diffusion.

$$
L_{\mu} / d \sim \frac{\sigma}{\mu_{1} U_{2}} \frac{(\mu \rho)^{1 / 3}}{\rho\left[1+(\mu \rho)^{1 / 3}\right]} .
$$

The equation for the surface velocity (12) is then valid only within a length $L_{\mu}$ from the jet source, but not downstream. After this length, one cannot use the dispersion relation (13) since the bulk velocities become inhomogeneous; in fact, the liquid bulk should become recirculating in order to accomplish the vanishing $Q_{1}$ condition (see Fig. 8). Naturally, other cases of UJ may exist when inertia and viscous forces are comparable [e.g., that sketched in Fig. 8(b)], but their required numerical treatment puts them outside the scope of the present analysis.

As a final remark, it is worth stressing the fundamental importance of the surface velocity $U_{s}$ in the present analysis, for both creeping and inertia-dominated flows: note that for the cases studied here, where analytical solutions can be found, the wave behavior with respect to an observer is strictly determined by the linearized kinematic condition at the interface:

$$
\frac{\partial R}{\partial t}+U_{s} \frac{\partial R}{\partial z}-V=0
$$

where $V$ is the small transverse component of the surface velocity. This is the only equation where the surface velocity appears; interestingly, since the first-order velocity profile does not enter into the analytical dispersion relations studied here [31], when the observer moves with the surface, its velocity disappears from the analysis, and one recovers the classical dispersion relations for cylindrical capillary columns existing in the literature.

\section{ACKNOWLEDGMENTS}

This work is supported by the Ministry of Science and Technology of Spain, Grant Nos. DPI2004-07197 and DPI2007-63559. Suggestions from Dr. Pascual RiescoChueca and discussions with Dr. José M. Montanero are highly appreciated. Dr. Miguel A. Herrada kindly supplied the unpublished data for the case $\mathrm{Re}_{D}=2200$ in Fig. 1 . 
[1] O. A. Basaran, AIChE J. 48, 1842 (2002).

[2] J. Zeleny, Phys. Rev. 10, 1 (1917).

[3] A. M. Gañán-Calvo, J. M. López-Herrera, and P. RiescoChueca, J. Fluid Mech. 566, 421 (2006).

[4] A. M. Gañán-Calvo, Phys. Rev. Lett. 98, 134503 (2007).

[5] J. M. Fernandez and G. M. Homsy, Phys. Fluids 16, 2548 (2004).

[6] A. Casner and J.-P. Delville, Phys. Rev. Lett. 90, 144503 (2003).

[7] S. P. Lin, Breakup of Liquid Sheets and Jets (Cambridge University Press, Cambridge, U.K., 2003).

[8] A. Sevilla, J. M. Gordillo, and C. Martinez-Bazan, Phys. Fluids 17, 018105 (2005).

[9] A. M. Gañán-Calvo and P. Riesco-Chueca, J. Fluid Mech. 553, 75 (2006).

[10] P. Guillot, A. Colin, A. S. Utada, and A. Ajdari, Phys. Rev. Lett. 99, 104502 (2007).

[11] A. M. Gañán-Calvo, Phys. Rev. Lett. 80, 285 (1998).

[12] P. M. Morse and H. Feshback, Methods of Theoretical Physics (McGraw-Hill, New York, 1953).

[13] A. M. Gañán-Calvo, Phys. Rev. E 75, 027301 (2007).

[14] A. M. Gañán-Calvo, R. González-Prieto, P. Riesco-Chueca, M. A. Herrada, and M. Flores-Mosquera, Nat. Phys. 3, 737 (2007).

[15] S. L. Anna, N. Bontoux, and H. A. Stone, Appl. Phys. Lett. 82, 364 (2003).

[16] R. Suryo and O. A. Basaran, Phys. Fluids 18, 082102 (2006).

[17] S. Courrech du Pont and J. Eggers, Phys. Rev. Lett. 96, 034501 (2006).

[18] S. L. Anna and H. C. Mayer, Phys. Fluids 18, 121512 (2006).
[19] T. R. Powers and R. E. Goldstein, Phys. Rev. Lett. 78, 2555 (1997).

[20] W. van Saarlos, Phys. Rev. A 37, 211 (1988).

[21] G. Dee and J. S. Langer, Phys. Rev. Lett. 50, 383 (1983).

[22] W. van Saarlos, Phys. Rev. Lett. 58, 2571 (1987).

[23] A. M. Gañán-Calvo, M. A. Herrada, and P. Garstecki, Phys. Rev. Lett. 96, 124504 (2006).

[24] A. S. Utada, A. Fernández-Nieves, H. A. Stone, and D. A. Weitz, Phys. Rev. Lett. 99, 094502 (2007).

[25] M. A. Herrada, A. M. Gañán-Calvo, and P. Guillot, e-print arXiv:0804.2586v2.

[26] A. M. Gañán-Calvo, M. Pérez-Saborid, J. M. López-Herrera, and J. M. Gordillo, Eur. Phys. J. B 39, 131 (2004).

[27] S. Anna (private communication).

[28] J. M. Montanero and A. M. Gañán-Calvo, Phys. Rev. E 77, 046301 (2008).

[29] W. W. Zhang, Phys. Rev. Lett. 93, 184502 (2004).

[30] The complex equation (13) provides six scalar equations from its real and imaginary terms of order $O(1), O(U)$, and $O\left(U^{2}\right)$. Conditions for $v_{-}^{*}=0$ provide three scalar equations resulting in $\operatorname{Im}\left(\omega_{0}\right)=0, \operatorname{Re}\left(\bar{\omega}_{1}\right)=0$, and $\operatorname{Im}\left(\omega_{1}\right)=0$.

[31] In the viscosity-dominated flow, the perturbed equations for both the pressure and the velocity are independent of the steady, first-order velocities (the convective term, the one responsible for a possible coupling between first order and perturbation, is absent). In the inertia-dominated flow considered here, since the basic velocity profiles are homogeneous, they enter the analysis only through the constant parameter $U=U_{2} / U_{1}$. 